\title{
Hawking radiation as tunneling from squashed Kaluza-Klein black hole
}

\author{
${ }^{1}$ Ken Matsuno* and ${ }^{2}$ Koichiro Umetsu ${ }^{\dagger}$ \\ ${ }^{1}$ Department of Mathematics and Physics, \\ Graduate School of Science, Osaka City University, \\ 3-3-138 Sugimoto, Sumiyoshi-ku, Osaka 558-8585, Japan \\ ${ }^{2}$ Maskawa Institute for Science and Culture, \\ Kyoto Sangyo University, Kyoto 603-8555, Japan
}

(Dated: October 27, 2018)

\begin{abstract}
We discuss Hawking radiation from a five-dimensional squashed Kaluza-Klein black hole on the basis of the tunneling mechanism. A simple manner, which was recently suggested by Umetsu, is possible to extend the original derivation by Parikh and Wilczek to various black holes. That is, we use the two-dimensional effective metric, which is obtained by the dimensional reduction near the horizon, as the background metric. By using same manner, we derive both the desired result of the Hawking temperature and the effect of the back reaction associated with the radiation in the squashed Kaluza-Klein black hole background.
\end{abstract}

PACS numbers: 04.50.-h, 04.70.Bw

\footnotetext{
* E-mail: matsuno@sci.osaka-cu.ac.jp

$\dagger$ E-mail: umetsu@cc.kyoto-su.ac.jp
} 


\section{INTRODUCTION}

In 1975, Hawking showed that a black hole can radiate from the event horizon like a blackbody at the temperature $T=\kappa /(2 \pi)$, where $\kappa$ is the surface gravity of the black hole, by using the method of quantum field theory in curved spacetime [1]. Thus Hawking radiation is one of interesting phenomena where both of general relativity and quantum theory play a role.

An elegant derivation of Hawking radiation on the basis of the tunneling method was proposed by Parikh and Wilczek [2]. The derivation of Hawking radiation on the basis of the tunneling effect has been discussed in the literature [3-76]. The essential idea of the tunneling mechanism is that a particle-antiparticle pair is formed close to the horizon inside a black hole. We can divide the field associated with a particle into ingoing modes moving toward the center of the black hole and outgoing modes trying to move outside the horizon. Then the ingoing modes are trapped inside the horizon, while a part of the outgoing modes escapes outside the black hole by the quantum tunneling effect. If the particle which comes out to our universe has positive energy, such a particle (and also an antiparticle) can stably exist and we can regard the particle outside the horizon as the radiation from the black hole. Parikh and Wilczek calculated the WKB probability amplitude for the particle by taking into account classically forbidden paths. By comparing the tunneling probability of an outgoing particle with the Boltzmann factor in thermodynamics, they successfully derived the Hawking temperature. The derivation on the basis of the tunneling method has an important advantage that it is possible to evaluate the back reaction associated with the radiation.

Parikh and Wilczek obtained above results for four-dimensional Schwarzschild and Reissner-Nordström black holes. The extensions of their derivation to four-dimensional Kerr and Kerr-Newman black holes have been discussed in [73 76]. The derivations for four-dimensional rotating black holes are mathematically complicated because of the effects of the rotation. Recently, one of the authors has suggested a new approach, which extends the method proposed by Parikh and Wilczek to four-dimensional rotating black holes, and showed that the derivation of the Hawking temperature is greatly simplified if one uses the technique of the dimensional reduction [77, 78]. This technique has been used in the derivation of Hawking radiation from anomalies and discussed for various black holes (for 
example, see $\left.\left[\begin{array}{ll}79 & 83\end{array}\right]\right)$. According to the technique, it is shown that metrics of various black holes effectively become two-dimensional spherically symmetric metrics near the horizon. We note that this technique of the dimensional reduction is valid only for the region very close to the horizon. Since the tunneling effect is a quantum one arising within the Planck length near the horizon region, the use of the technique of the dimensional reduction in the tunneling mechanism is justified. It is thus possible to naturally extend the method of Parikh and Wilczek to cases of various black holes and we can derive both the Hawking temperatures and the effects of the back reactions associated with the radiations in various black hole backgrounds by using the new approach. Then the derivations of the Hawking temperatures from four-dimensional rotating black holes become simple in comparison with previous ones [73-76].

A family of squashed Kaluza-Klein black holes is one of interesting solutions of higherdimensional black holes. In the context of string theory and brane world scenario, investigations on higher-dimensional black hole solutions have attracted a lot of attention. From a realistic point of view, the extra dimensions need to be compactified to reconcile the higher-dimensional theory of gravity with our apparently four-dimensional world. We consider higher-dimensional black holes in the spacetime with compact extra dimensions, i.e., Kaluza-Klein black holes. In higher-dimensional spacetimes, even if we impose asymptotic flatness to the four-dimensional part of the spacetime, there are various possibilities of fiber bundle structures of the extra dimensions as the fiber over the four-dimensional base spacetime. The black hole solutions with nontrivial bundle structures have been studied by various authors. Dobiasch and Maison found the first five-dimensional vacuum Kaluza-Klein black hole solution which asymptotes to a twisted $S^{1}$ fiber bundle over the four-dimensional Minkowski spacetime [84], and Gibbons and Wiltshire clarified the meaning of the metric [85]. Later, the solution was generalized to the charged case in five-dimensional EinsteinMaxwell theory [86]. It is shown that this family of black holes has squashed $S^{3}$ horizons, and that the metrics behave as fully five-dimensional black holes in the vicinity of the horizon, while they behave as four-dimensional black holes in the region far away from the horizon. That is, the squashed Kaluza-Klein black hole solutions asymptote to locally flat spacetimes with a compactified extra dimension and we can regard a series of these solutions as one of realistic higher-dimensional black hole models. Several aspects of squashed Kaluza-Klein black holes are also discussed, for example, thermodynamics [87 89], Hawking radiation 
[90 92], quasinormal modes [93 95], stabilities [96, 97], geodetic precession [98] and strong gravitational lensing [99].

In this paper, we consider the Hawking radiation from a five-dimensional charged static Kaluza-Klein black hole with squashed horizons on the basis of the tunneling mechanism. To the best our knowledge, derivations of Hawking radiation in the framework of the tunneling method have not been discussed in asymptotically Kaluza-Klein spacetimes. In the present work, we extend the derivation of Hawking radiation as a tunneling process by using the technique of the dimensional reduction near the horizon in four dimensions to the case of the five-dimensional squashed Kaluza-Klein black hole. For simplicity, we restrict ourselves to uncharged radiations coming from the black hole and take into account the back reaction of the radiation. Then, in contrast to the previous studies [90 92], we obtain not only the Hawking temperature of the black hole but also the effect of the back reaction associated with the radiation in a very simple manner.

This paper is organized as follows. In Section II, we review the properties of fivedimensional charged static Kaluza-Klein black hole solutions with squashed horizons. In Section III, we show that the five-dimensional squashed Kaluza-Klein black hole metric effectively becomes a two-dimensional spherically symmetric metric by using the technique of the dimensional reduction near the horizon. In Section IV] we apply the tunneling mechanism to the squashed Kaluza-Klein black hole and derive both the Hawking temperature and the effect of the back reaction associated with the radiation. Section $\nabla$ is devoted to discussion and conclusion.

\section{REVIEW OF SQUASHED KALUZA-KLEIN BLACK HOLES}

We consider the charged static Kaluza-Klein black hole with squashed $S^{3}$ horizons, which is one of the exact solutions of the five-dimensional Einstein-Maxwell theory [86]. The metric and the gauge potential are written as

$$
\begin{aligned}
d s^{2} & =-F(\rho) d t^{2}+\frac{K^{2}(\rho)}{F(\rho)} d \rho^{2}+\rho^{2} K^{2}(\rho) d \Omega_{S^{2}}^{2}+\frac{r_{\infty}^{2}}{4 K^{2}(\rho)}(d \psi+\cos \theta d \phi)^{2} \\
A_{\mu} & =\left( \pm \frac{\sqrt{3 \rho_{+} \rho_{-}}}{2 \rho}, 0,0,0,0\right)
\end{aligned}
$$


where $d \Omega_{S^{2}}^{2}=d \theta^{2}+\sin ^{2} \theta d \phi^{2}$ denotes the metric of the unit two-sphere, and the functions $F(\rho)$ and $K(\rho)$ are given by

$$
F(\rho)=\frac{\rho^{2}-2 M \rho+Q^{2}}{\rho^{2}}=\frac{\left(\rho-\rho_{+}\right)\left(\rho-\rho_{-}\right)}{\rho^{2}}, \quad K^{2}(\rho)=\frac{\rho+\rho_{0}}{\rho} .
$$

The parameters $r_{\infty}, \rho_{ \pm}$, and $\rho_{0}$ are related as $r_{\infty}^{2}=4\left(\rho_{+}+\rho_{0}\right)\left(\rho_{-}+\rho_{0}\right)$, and the parameters $M$ and $Q$ denote the Komar mass and the charge of the black hole (1), respectively. The coordinates $(t, \rho, \theta, \phi, \psi)$ run the ranges of $-\infty<t<\infty, 0<\rho<\infty, 0 \leq \theta \leq \pi, 0 \leq$ $\phi \leq 2 \pi$, and $0 \leq \psi \leq 4 \pi$, respectively. To avoid the existence of naked singularities on and outside the horizon, we choose the parameters such that $\rho_{+} \geq \rho_{-} \geq 0$ and $\rho_{-}+\rho_{0}>0$.

The outer and the inner horizons are located at $\rho=\rho_{+}=M+\sqrt{M^{2}-Q^{2}}$ and $\rho=\rho_{-}=$ $M-\sqrt{M^{2}-Q^{2}}$, respectively. The shapes of horizons are the squashed $S^{3}$ in the form of the Hopf bundle. The surface gravity on the outer horizon of the black hole (11) is obtained as

$$
\kappa=\frac{\rho_{+}-\rho_{-}}{2 \rho_{+}^{2}} \sqrt{\frac{\rho_{+}}{\rho_{+}+\rho_{0}}} .
$$

At infinity, $\rho \rightarrow \infty$, the metric (1) behaves as

$$
d s^{2} \simeq-d t^{2}+d \rho^{2}+\rho^{2} d \Omega_{S^{2}}^{2}+\frac{r_{\infty}^{2}}{4}(d \psi+\cos \theta d \phi)^{2} .
$$

That is, the metric (11) asymptotes to a twisted constant $S^{1}$ fiber bundle over the fourdimensional Minkowski spacetime. The size of the compactified extra dimension of the spacetime (1) at infinity is given by $r_{\infty}$.

Here, we discuss the physical meanings of the parameter $\rho_{0}$ in the metric (11). If $\rho_{0} \ll \rho_{ \pm}$, $\rho$ dependence of the function $F(\rho)$ is important but the function $K(\rho)$ is almost unity for an observer outside the horizons, $\rho_{0} \ll \rho_{ \pm} \lesssim \rho$. Then, the observer feels the spacetime as the four-dimensional Reissner-Nordström black hole with a twisted constant $S^{1}$ fiber with the metric

$$
d s^{2} \simeq-\frac{\left(\rho-\rho_{+}\right)\left(\rho-\rho_{-}\right)}{\rho^{2}} d t^{2}+\frac{\rho^{2}}{\left(\rho-\rho_{+}\right)\left(\rho-\rho_{-}\right)} d \rho^{2}+\rho^{2} d \Omega_{S^{2}}^{2}+\frac{r_{\infty}^{2}}{4}(d \psi+\cos \theta d \phi)^{2} .
$$

On the other hand, if $\rho_{ \pm} \ll \rho_{0}$, the function $K(\rho)$ becomes important for an observer at $\rho_{ \pm} \lesssim \rho \ll \rho_{0}$. With the help of a new coordinate $r=2 \sqrt{\rho_{0} \rho}$ and parameters $r_{ \pm}=2 \sqrt{\rho_{0} \rho_{ \pm}}$, since $r_{ \pm}^{2} \ll r_{\infty}^{2}$, the metric (11) approaches the five-dimensional Reissner-Nordström black hole [100]:

$$
d s^{2} \simeq-\frac{\left(r^{2}-r_{+}^{2}\right)\left(r^{2}-r_{-}^{2}\right)}{r^{4}} d t^{2}+\frac{r^{4}}{\left(r^{2}-r_{+}^{2}\right)\left(r^{2}-r_{-}^{2}\right)} d r^{2}+r^{2} d \Omega_{S^{3}}^{2},
$$


where $d \Omega_{S^{3}}^{2}$ denotes the metric of the unit three-sphere. Then, the observer feels the spacetime as an almost $S^{3}$ symmetric black hole. Therefore, the parameter $\rho_{0}$ gives the typical scale of transition from five dimensions to effective four dimensions.

\section{DIMENSIONAL REDUCTION NEAR THE HORIZON}

In this section, we discuss the technique of the dimensional reduction near the horizon in the squashed Kaluza-Klein black hole. Considering the near horizon behavior of the action for a five-dimensional complex scalar field in the squashed Kaluza-Klein black hole background (1), we show that the five-dimensional action is reduced to a two-dimensional one and the five-dimensional squashed Kaluza-Klein black hole behaves as an effectively two-dimensional black hole in the region very close to the horizon.

We consider the five-dimensional complex scalar field $\Phi$ in the squashed Kaluza-Klein black hole background (1). The action is given by

$$
S=\int d^{5} x \sqrt{-g} g^{\mu \nu}\left(\partial_{\mu}+i e A_{\mu}\right) \Phi^{*}\left(\partial_{\nu}-i e A_{\nu}\right) \Phi+S_{\mathrm{int}},
$$

where the first term is the kinetic term and the second term $S_{\text {int }}$ represents the mass, the potential and the interaction terms. Substituting the metric (11) and the gauge potential (2) into the action (8), we have

$$
\begin{aligned}
S= & -\frac{r_{\infty}}{2} \int d t d \rho d \theta d \phi d \psi \sin \theta \rho^{2} K^{2} \Phi^{*}\left[-\frac{1}{F}\left(\partial_{t}-i e A_{t}\right)^{2}+\frac{1}{\rho^{2} K^{2}} \partial_{\rho}\left(\rho^{2} F \partial_{\rho}\right)\right. \\
& \left.+\frac{1}{\rho^{2} K^{2} \sin \theta} \partial_{\theta}\left(\sin \theta \partial_{\theta}\right)+\frac{1}{\rho^{2} K^{2}}\left(\frac{1}{\sin \theta} \partial_{\phi}-\frac{\cos \theta}{\sin \theta} \partial_{\psi}\right)^{2}+\frac{4 K^{2}}{r_{\infty}^{2}} \partial_{\psi}^{2}\right] \Phi+S_{\mathrm{int}} .
\end{aligned}
$$

We perform the partial wave decomposition of $\Phi$ as

$$
\Phi=\sum_{l, m, \lambda} \Phi_{l m \lambda}(t, \rho) S_{l m \lambda}(\theta) e^{i(m \phi+\lambda \psi)}
$$

where the function $S_{\operatorname{lm} \lambda}$ is the spin-weighted spherical function, which satisfies

$$
\left[\frac{1}{\sin \theta} \partial_{\theta}\left(\sin \theta \partial_{\theta}\right)-\frac{(m-\lambda \cos \theta)^{2}}{\sin ^{2} \theta}+l(l+1)-\lambda^{2}\right] S_{l m \lambda}=0 .
$$

We have the conditions such that $l \geq \lambda$ and $m=-l,-l+1, \cdots, l$. The periodicity also 
requires that $2 \lambda, 2 m$, and $\lambda \pm m$ are integers. Then we obtain the action (9) in the form

$$
\begin{aligned}
S= & -\frac{r_{\infty}}{2} \int d t d \rho d \theta d \phi d \psi \sin \theta \rho^{2} K^{2} \sum_{l^{\prime}, m^{\prime}, \lambda^{\prime}} \Phi_{l^{\prime} m^{\prime} \lambda^{\prime}}^{*} S_{l^{\prime} m^{\prime} \lambda^{\prime}}^{*} e^{-i\left(m^{\prime} \phi+\lambda^{\prime} \psi\right)} \\
& \times\left[-\frac{1}{F}\left(\partial_{t}-i e A_{t}\right)^{2}+\frac{1}{\rho^{2} K^{2}} \partial_{\rho}\left(\rho^{2} F \partial_{\rho}\right)-\frac{l(l+1)-\lambda^{2}}{\rho^{2} K^{2}}-\frac{4 K^{2} \lambda^{2}}{r_{\infty}^{2}}\right] \\
& \times \sum_{l, m, \lambda} \Phi_{l m \lambda} S_{l m \lambda} e^{i(m \phi+\lambda \psi)}+S_{\mathrm{int}} .
\end{aligned}
$$

Define the tortoise coordinate as

$$
d \rho_{*}=\frac{K}{F} d \rho,
$$

the action (12) is rewritten as

$$
\begin{aligned}
S= & -\frac{r_{\infty}}{2} \int d t d \rho_{*} d \theta d \phi d \psi \sin \theta \rho^{2} K \sum_{l^{\prime}, m^{\prime}, \lambda^{\prime}} \Phi_{l^{\prime} m^{\prime} \lambda^{\prime}}^{*} S_{l^{\prime} m^{\prime} \lambda^{\prime}}^{*} e^{-i\left(m^{\prime} \phi+\lambda^{\prime} \psi\right)} \\
& \times\left[-\left(\partial_{t}-i e A_{t}\right)^{2}+\frac{1}{\rho^{2} K} \partial_{\rho_{*}}\left(\rho^{2} K \partial_{\rho_{*}}\right)-F\left\{\frac{l(l+1)-\lambda^{2}}{\rho^{2} K^{2}}+\frac{4 K^{2} \lambda^{2}}{r_{\infty}^{2}}\right\}\right] \\
& \times \sum_{l, m, \lambda} \Phi_{l m \lambda} S_{l m \lambda} e^{i(m \phi+\lambda \psi)}+S_{\mathrm{int}} .
\end{aligned}
$$

We consider the action (14) in the region near the black hole horizon, $\rho \simeq \rho_{+}$. Since the theory becomes the high-energy theory near the horizon and $F\left(\rho_{+}\right)=0$ at $\rho \rightarrow \rho_{+}$, we ignore all terms in $S_{\text {int }}$ and retain the dominant kinetic term only. Then we have

$$
\begin{aligned}
S= & -\frac{r_{\infty}}{2} \int d t d \rho_{*} d \theta d \phi d \psi \sin \theta \rho^{2} K \sum_{l^{\prime}, m^{\prime}, \lambda^{\prime}} \Phi_{l^{\prime} m^{\prime} \lambda^{\prime}}^{*} S_{l^{\prime} m^{\prime} \lambda^{\prime}}^{*} e^{-i\left(m^{\prime} \phi+\lambda^{\prime} \psi\right)} \\
& \times\left[-\left(\partial_{t}-i e A_{t}\right)^{2}+\frac{1}{\rho^{2} K} \partial_{\rho_{*}}\left(\rho^{2} K \partial_{\rho_{*}}\right)\right] \sum_{l, m, \lambda} \Phi_{l m \lambda} S_{l m \lambda} e^{i(m \phi+\lambda \psi)} .
\end{aligned}
$$

We return to the expression written in terms of the coordinate $\rho$, the action (15) takes the form

$$
S=-4 \pi^{2} r_{\infty} \sum_{l, m, \lambda} \int d t d \rho \rho^{2} K \Phi_{l m \lambda}^{*}\left[-\frac{K}{F}\left(\partial_{t}-i e A_{t}\right)^{2}+\partial_{\rho}\left(\frac{F}{K} \partial_{\rho}\right)\right] \Phi_{l m \lambda}
$$

where we have used the orthonormal condition for the spin-weighted spherical function,

$$
\int d \theta \sin \theta S_{l^{\prime} m^{\prime} \lambda^{\prime}}^{*} S_{l m \lambda}=\delta_{l^{\prime}, l} \delta_{m^{\prime}, m} \delta_{\lambda^{\prime}, \lambda}
$$

and integrated the angular coordinate parts. From the action (16), we can regard the field $\Phi_{l m \lambda}$ as a two-dimensional complex scalar field in the backgrounds with the metric $d s_{2 \mathrm{D}}^{2}$, the 
gauge potential $A_{i}(i=t, \rho)$, and the dilaton field $\Psi$ :

$$
\begin{aligned}
& d s_{2 \mathrm{D}}^{2}=-\frac{F}{K} d t^{2}+\frac{K}{F} d \rho^{2}, \\
& A_{t}= \pm \frac{\sqrt{3 \rho_{+} \rho_{-}}}{2 \rho}, \quad A_{\rho}=0 \\
& \Psi=\rho^{2} K
\end{aligned}
$$

where the functions $F$ and $K$ are given by the equations (3).

We see that the metric of the five-dimensional squashed Kaluza-Klein black hole (1) behaves as an effectively two-dimensional metric (18) by using the technique of the dimensional reduction near the horizon. For confirmation, we calculate the surface gravity on the horizon of the two-dimensional spacetime (18) as

$$
\kappa=\left.\frac{1}{2} \partial_{\rho}\left(\frac{F}{K}\right)\right|_{\rho=\rho_{+}}=\frac{\rho_{+}-\rho_{-}}{2 \rho_{+}^{2}} \sqrt{\frac{\rho_{+}}{\rho_{+}+\rho_{0}}} .
$$

We see that this surface gravity (21) coincides with that of the five-dimensional squashed Kaluza-Klein black hole (4).

\section{HAWKING RADIATION AS TUNNELING PROCESS}

In this section, we derive both the Hawking temperature and the effect of the back reaction associated with the radiation in the squashed Kaluza-Klein black hole background on the basis of the tunneling mechanism. Here we use the two-dimensional effective metric (18) as the background metric. Since the tunneling effect is a quantum one arising within the Planck length near the horizon region, our derivation of Hawking radiation as a tunneling process by using the technique of the dimensional reduction is justified.

To describe the across-horizon phenomena, Parikh and Wilczek used the Painlevé coordinates, where the coordinate singularity at the horizon was removed [2]. Similarly, we define the Painlevé-like coordinate $t_{p}$ to the metric (18) by

$$
d t_{p}=d t+\frac{K}{F} \sqrt{1-\frac{F}{K}} d \rho .
$$

Then the metric (18) is rewritten as

$$
d s_{2 \mathrm{D}}^{2}=-\frac{\rho^{2}-2 M \rho+Q^{2}}{\rho^{2} K} d t_{p}^{2}+2 \sqrt{\frac{K-1}{K}+\frac{2 M}{\rho K}-\frac{Q^{2}}{\rho^{2} K}} d t_{p} d \rho+d \rho^{2} .
$$


The metric (23) has a number of interesting features. At any fixed time, $t_{p}=$ const., the spatial geometry is flat, and for any fixed radius, $\rho=$ const., the boundary geometry is the same as that of the metric (18).

The radial null geodesics for the metric (23) are given by $d s_{2 \mathrm{D}}^{2}=0$. We obtain

$$
\dot{\rho}=\frac{d \rho}{d t_{p}}= \pm 1-\sqrt{\frac{K-1}{K}+\frac{2 M}{\rho K}-\frac{Q^{2}}{\rho^{2} K}}
$$

where the upper and the lower signs correspond to the outgoing and the ingoing geodesics, respectively, under the implicit assumption that $t_{p}$ increases towards the future. Since we can ignore the mass of matter field near the horizon region by the discussion of dimensional reduction, the use of null geodesics (24) in our derivation of Hawking radiation is justified. If we restrict ourselves to uncharged radiations coming from the black hole and take into account the effect of the particle's self gravitation, i.e., the back reaction of the radiation, we replace the mass of the black hole $M$ by $M-\omega$, where $\omega$ denotes the energy of the particle which escapes from the black hole by the tunneling mechanism. Then we have the metric (23) and the radial null geodesics (24) in the forms

$$
\begin{aligned}
d s_{2 \mathrm{D}}^{2} & =-\frac{\rho^{2}-2(M-\omega) \rho+Q^{2}}{\rho^{2} K} d t_{p}^{2}+2 \sqrt{\frac{K-1}{K}+\frac{2(M-\omega)}{\rho K}-\frac{Q^{2}}{\rho^{2} K}} d t_{p} d \rho+d \rho^{2}, \\
\dot{\rho} & = \pm 1-\sqrt{\frac{K-1}{K}+\frac{2(M-\omega)}{\rho K}-\frac{Q^{2}}{\rho^{2} K}} .
\end{aligned}
$$

By following the method proposed by Parikh and Wilczek, we evaluate the WKB probability amplitude for a classically forbidden trajectory. The imaginary part of the action for an outgoing positive energy particle, which crosses the horizon outwards from $\rho=\rho_{\text {in }}$ to $\rho=\rho_{\text {out }}$, is given by

$$
\operatorname{Im} S=\operatorname{Im} \int_{\rho_{\text {in }}}^{\rho_{\text {out }}} p_{\rho} d \rho=\operatorname{Im} \int_{\rho_{\text {in }}}^{\rho_{\text {out }}} \int_{0}^{p_{\rho}} d p_{\rho}^{\prime} d \rho=\operatorname{Im} \int_{\rho_{\text {in }}}^{\rho_{\text {out }}} \int_{0}^{H} \frac{d H^{\prime}}{\dot{\rho}} d \rho,
$$

where we have multiplied and divided the integrand by the two sides of Hamilton's equation, $\dot{\rho}=d H /\left.d p_{\rho}\right|_{\rho}$, to change the variable from the momentum to the energy. Taking into account the effect of the back reaction of the radiation, we obtain

$$
\operatorname{Im} S=\operatorname{Im} \int_{M}^{M-\omega} \int_{\rho_{\text {in }}}^{\rho_{\text {out }}} \frac{d \rho}{\dot{\rho}} d H^{\prime}=\operatorname{Im} \int_{0}^{\omega} \int_{\rho_{\text {in }}}^{\rho_{\text {out }}} \frac{d \rho}{1-\sqrt{\frac{K-1}{K}+\frac{2\left(M-\omega^{\prime}\right)}{\rho K}-\frac{Q^{2}}{\rho^{2} K}}}\left(-d \omega^{\prime}\right),
$$

where we have used the Hamiltonian, $H=M-\omega$, and the radial null geodesic (26) with the upper sign. Now the integral can be done by deforming the contour, so as to ensure 
that positive energy solutions decay in time, that is, into the lower half $\omega^{\prime}$ plane. By using Feynman's $i \epsilon$ prescription, $\omega \rightarrow \omega-i \epsilon$, we have

$$
\begin{aligned}
\operatorname{Im} S & =\operatorname{Im} \int_{\rho_{\text {in }}}^{\rho_{\text {out }}} \int_{M}^{M-\omega} \frac{d M^{\prime}}{1-\sqrt{\frac{K-1}{K}+\frac{2 M^{\prime}}{\rho K}-\frac{Q^{2}}{\rho^{2} K}}} d \rho \\
& =\operatorname{Im} \int_{\rho_{\text {in }}}^{\rho_{\text {out }}} \int_{M}^{M-\omega} \frac{d M^{\prime}}{1-\sqrt{\frac{K-1}{K}+\frac{2 M^{\prime}}{\rho K}-\frac{Q^{2}}{\rho^{2} K}}+i \epsilon} d \rho \\
& =\operatorname{Im} \int_{\rho_{\text {in }}}^{\rho_{\text {out }}} \int_{M}^{M-\omega}-i \pi \delta\left(1-\sqrt{\frac{K-1}{K}+\frac{2 M^{\prime}}{\rho K}-\frac{Q^{2}}{\rho^{2} K}}\right) d M^{\prime} d \rho \\
& =-\pi \int_{\rho_{\text {in }}}^{\rho_{\text {out }}} \rho K d \rho .
\end{aligned}
$$

Thus we obtain the WKB probability amplitude as

$$
\begin{aligned}
\Gamma \simeq & e^{-2 \operatorname{Im} S} \\
= & \exp \left[\frac{\pi}{2}\left(2 \rho_{\text {out }}+\rho_{0}\right) \sqrt{\rho_{\text {out }}\left(\rho_{\text {out }}+\rho_{0}\right)}-\frac{\pi}{2}\left(2 \rho_{\text {in }}+\rho_{0}\right) \sqrt{\rho_{\text {in }}\left(\rho_{\text {in }}+\rho_{0}\right)}\right. \\
& \left.-\frac{\pi \rho_{0}^{2}}{2} \log \frac{\sqrt{\rho_{\text {out }}}+\sqrt{\rho_{\text {out }}+\rho_{0}}}{\sqrt{\rho_{\text {in }}}+\sqrt{\rho_{\text {in }}+\rho_{0}}}\right],
\end{aligned}
$$

where the explicit forms of $\rho_{\text {in }}$ and $\rho_{\text {out }}$ are, respectively, given by

$$
\rho_{\text {in }}=\rho_{+}=M+\sqrt{M^{2}-Q^{2}}, \quad \rho_{\text {out }}=M-\omega+\sqrt{(M-\omega)^{2}-Q^{2}} .
$$

If we consider the effect in the second order of $\omega$, in terms of $\rho_{+}$and $\rho_{-}$, the WKB probability amplitude (30) takes the form

$$
\Gamma \simeq \exp \left(-\frac{2 \pi}{\tilde{\kappa}} \omega+\alpha \omega^{2}\right)
$$

where the constants $\tilde{\kappa}$ and $\alpha$ are, respectively, given by

$$
\begin{aligned}
\tilde{\kappa} & =\frac{\rho_{+}-\rho_{-}}{2 \rho_{+}^{2}} \sqrt{\frac{\rho_{+}}{\rho_{+}+\rho_{0}}}, \\
\alpha & =\frac{2 \pi \rho_{+}\left[2 \rho_{+}\left(\rho_{+}-3 \rho_{-}\right)+\rho_{0}\left(\rho_{+}-5 \rho_{-}\right)\right]}{\left(\rho_{+}-\rho_{-}\right)^{3}} \sqrt{\frac{\rho_{+}}{\rho_{+}+\rho_{0}}} .
\end{aligned}
$$

We see that the constant $\tilde{\kappa}(33)$ coincides with the surface gravity of the black hole (4), that is, $\tilde{\kappa}=\kappa$. Thus, by comparing the WKB probability amplitude (32) to the first order in $\omega$ with the Boltzmann factor in a thermal equilibrium state at the temperature $T, \Gamma=e^{-\omega / T}$, we obtain the Hawking temperature of the five-dimensional squashed Kaluza-Klein black hole (11) as

$$
T_{\mathrm{H}}=\frac{\tilde{\kappa}}{2 \pi}=\frac{\rho_{+}-\rho_{-}}{4 \pi \rho_{+}^{2}} \sqrt{\frac{\rho_{+}}{\rho_{+}+\rho_{0}}},
$$


which is the desired result [87 90, 92], and we can regard the quadratic term of $\omega$ in the equation (32), $\alpha \omega^{2}$, as the correction by the back reaction of the radiation.

In the limit, $\rho_{0} \rightarrow 0$, we obtain the metric (11) with $K=1$, which locally has the geometry of the black string (6). In this limit, the WKB probability amplitude (30) reduces to

$$
\begin{aligned}
\Gamma & \simeq \exp \left[\pi\left(\rho_{\text {out }}^{2}-\rho_{\text {in }}^{2}\right)\right] \\
& =\exp \left[-4 \pi \omega\left(M-\frac{\omega}{2}\right)+2 \pi(M-\omega) \sqrt{(M-\omega)^{2}-Q^{2}}-2 \pi M \sqrt{M^{2}-Q^{2}}\right] .
\end{aligned}
$$

To the first order in $\omega$, this WKB probability amplitude (36) is consistent with previous results of thermal emission at the Hawking temperature $T_{\mathrm{H}}$ for the four-dimensional ReissnerNordström black hole [1, 2]:

$$
T_{\mathrm{H}}=\frac{\sqrt{M^{2}-Q^{2}}}{2 \pi\left(M+\sqrt{M^{2}-Q^{2}}\right)^{2}} .
$$

\section{DISCUSSION AND CONCLUSION}

To sum up, we have extended the derivation of Hawking radiation by using the tunneling mechanism in four-dimensional black hole backgrounds to the case of the five-dimensional charged static Kaluza-Klein black hole with squashed horizons. We have restricted ourselves to uncharged radiations coming from the black hole and taken into account the back reaction of the radiation. Then we have derived the Hawking temperature of the black hole in a very simple manner. The calculation in our derivation by using the technique of the dimensional reduction near the horizon is little different from the original calculation for four-dimensional spherically symmetric black holes discussed in [2]. Although, our method has included the essential effect of the extra dimension, we have derived the Hawking temperature correctly by evaluating the effect to the first order in the energy of the particle which escapes from the black hole by the tunneling mechanism. We have seen that the technique of the dimensional reduction has been useful in the derivation of Hawking radiation on the basis of the tunneling mechanism in the five-dimensional squashed Kaluza-Klein black hole backgrounds. In contrast to the previous studies of Hawking radiation from squashed Kaluza-Klein black holes [90 92], we have derived not only the Hawking temperature but also the effect of the back reaction associated with the radiation. If higher-dimensional black holes are created in future accelerator experiments and we assume that the five-dimensional squashed Kaluza-

Klein black hole solutions describe geometries around such black holes, we expect that our 
present work could make a contribution to the verifications of Hawking radiation and extra dimensions in asymptotically Kaluza-Klein spacetimes.

In this paper, our analysis by using the tunneling mechanism has been confined only to the derivation of the Hawking temperature of the five-dimensional squashed Kaluza-Klein black hole and we have not discussed the blackbody spectrum. Then there remains the possibility that the black hole is not the blackbody but merely the thermal body. However, we expect that we can obtain the blackbody spectrum of the Hawking radiation in the squashed Kaluza-Klein black hole background by applying the methods in [77, 101, 102], where the derivation of the blackbody spectrum with the Hawking temperature from the expectation value of number operator by using the properties of the tunneling mechanism is discussed. We leave the analysis of the blackbody spectrum for the five-dimensional squashed Kaluza-Klein black hole on the basis of the tunneling mechanism for the future.

[1] S. W. Hawking, Commun. Math. Phys. 43, 199 (1975) [Erratum-ibid. 46, 206 (1976)].

[2] M. K. Parikh and F. Wilczek, Phys. Rev. Lett. 85, 5042 (2000) arXiv:hep-th/9907001.

[3] K. Srinivasan and T. Padmanabhan, Phys. Rev. D60, 024007 (1999) arXiv:gr-qc/9812028.

[4] M.K.Parikh Int. J. Mod. Phys. D13, 2351 (2004) arXiv:hep-th/0405160].

[5] S. Shankaranarayanan, K. Srinivasan and T. Padmanabhan, Mod. Phys. Lett. A16, 571 (2001) arXiv:gr-qc/0007022.

[6] S. Shankaranarayanan, T. Padmanabhan, and K. Srinivasan, Class. Quant. Grav. 19, 2671 (2002) arXiv:gr-qc/0010042.

[7] S. Shankaranarayanan, Phys. Rev. D67, 084026 (2003) [arXiv:gr-qc/0301090].

[8] E.C.Vagenas, Nuovo Cim. B117, 899 (2002) arXiv:hep-th/0111047).

[9] M. Angheben, M. Nadalini, L. Vanzo and S. Zerbini, JHEP 0505, 014 (2005) arXiv:hep-th/0503081].

[10] M. Arzano, A. J. M. Medved and E. C. Vagenas, JHEP 0509, 037 (2005) arXiv:hep-th/0505266.

[11] A. J. M. Medved and E. C. Vagenas, Mod. Phys. Lett. A20, 2449 (2005) arXiv:gr-qc/0504113].

[12] Q. Q. Jiang and S. Q. Wu, Phys. Lett. B635, 151 (2006) arXiv:hep-th/0511123. 
[13] D. Y. Chen, Q. Q. Jiang, S. Z. Yang and X. T. Zu, Class.Quant.Grav. 25205022 (2008) arXiv:0803.3248.

[14] D. Y. Chen, Q. Q. Jiang and X. T. Zu, Phys.Lett. B665, 106 (2008) [arXiv:0804.0131].

[15] Q. Q. Jiang, Phys. Rev. D78, 044009 (2008) arXiv:0807.1358.

[16] Q. Q. Jiang, Phys. Lett. B666, 517 (2008) [arXiv:].

[17] E. T. Akhmedov, V. Akhmedova and D. Singleton, Phys. Lett. B642, 124 (2006) arXiv:hep-th/0608098.

[18] E. T. Akhmedov, V. Akhmedova, D. Singleton and T. Pilling, Int. J. Mod. Phys. A22, 1705 (2007) arXiv:hep-th/0605137.

[19] E. T. Akhmedhov, T. Pilling, D. Singleton, Int. J. Mod. Phys. D17, 2453 (2008) arXiv:0805.2653].

[20] Y. Hu, J. Zhang and Z. Zhao, Mod. Phys. Lett. A21 2143 (2006) arXiv:gr-qc/0611026.

[21] Y. Hu, J. Zhang and Z. Zhao, Int. J. Mod. Phys. D16, 847 (2007) arXiv:gr-qc/0611085.

[22] Y. P. Hu, J. Y. Zhang and Z. Zhao, arXiv:0901.2680.

[23] P.Mitra, Phys. Lett. B648, 240 (2007) arXiv:hep-th/0611265.

[24] C. Z. Liu and J. Y. Zhu, Gen. Rel. Grav. 40, 1899 (2008) arXiv:gr-qc/0703055.

[25] B.D.Chowdhury, Pramana 70, 593 (2008) arXiv:hep-th/0605197].

[26] T.Pilling, Phys. Lett. B 660, 402 (2008) arXiv:0709.1624.

[27] S. P. Kim, J. Korean Phys. Soc. 53, 1095 (2008) arXiv:0709.4313.

[28] S. P. Kim, JHEP 0711, 048 (2007) arXiv:0710.0915].

[29] S. Sarkar and D. Kothawala, Phys. Lett. B659, 683 (2008) arXiv:0709.4448.

[30] R. Kerner and R. B. Mann, Class. Quant. Grav. 25, 095014 (2008) arXiv:0710.0612].

[31] R. Banerjee, B. R. Majhi, Phys. Lett. B662, (2008) 62 [arXiv:0801.0200.

[32] R. Banerjee, B. R. Majhi and S. Samanta, Phys. Rev. D77, 124035 (2008) arXiv:0801.3583.

[33] R. Banerjee and B. R. Majhi, JHEP 0806, 095 (2008) arXiv:0805.2220.

[34] S. K. Modak, Phys. Lett. B671, 167 (2009) arXiv:0807.0959].

[35] R. Banerjee and B. R. Majhi, Phys. Lett. B674, 218 (2009) arXiv:0808.3688.

[36] B. R. Majhi, Phys. Rev. D79, 044005 (2009) [arXiv:0809.1508].

[37] R. Banerjee, B. R. Majhi, Phys. Rev. D79, 064024, (2009) arXiv:0812.0497.

[38] R. Banerjee, B. R. Majhi, D. Roy, arXiv:0901.0466.

[39] B. R. Majhi and S. Samanta, Annals Phys. 325, 2410 (2010) [arXiv:0901.2258 [hep-th]]. 
[40] R. Li and J. R. Ren, Phys. Lett. B661, 370 (2008) arXiv:0802.3954].

[41] R. Li and J. R. Ren, Class. Quant. Grav. 25125016 (2008) arXiv:0803.1410.

[42] R. Li, J. R. Ren and D. F. Shi, Phys.Lett. B670, 446 (2009) [arXiv:0812.4217].

[43] R. D. Criscienzo and L. Vanzo, Europhys. Lett. 82, 60001 (2008) arXiv:0803.0435].

[44] R. Kerner and R. B. Mann, Phys. Lett. B665, 277 (2008) arXiv:0803.2246.

[45] G. E. Volovik, Int. J. Mod. Phys. D 18, 1227 (2009) arXiv:0803.3367 [hep-th]].

[46] J. Zhang, Phys.Lett. B668, 353 (2008) arXiv:0806.2441].

[47] S. Stotyn, K. Schleich and D. Witt, Class. Quant. Grav. 26, 065010 (2009) arXiv:0809.5093.

[48] H. M. Siahaan and Triyanta, arXiv:0811.1132.

[49] T. Zhu and J. R. Ren, Eur. Phys. J. C 62, 413 (2009) arXiv:0811.4074 [hep-th]].

[50] K. Chiang, S. M. Ke, D. T. Peng and F. Tsun, arXiv:0812.3006.

[51] R. Banerjee and S. K. Modak, JHEP 05, 063 (2009) arXiv:0903.3321.

[52] H. M. Siahaan, arXiv:0903.3723.

[53] X. n. Wu and S. Gao, Phys. Rev. D 75, 044027 (2007) arXiv:gr-qc/0702033.

[54] G. E. Volovik, Pisma Zh. Eksp. Teor. Fiz. 69, 662 (1999) [JETP Lett. 69, 705 (1999)] arXiv:gr-qc/9901077].

[55] E. C. Vagenas, Phys. Lett. B 503, 399 (2001) [arXiv:hep-th/0012134].

[56] E. C. Vagenas, Mod. Phys. Lett. A 17, 609 (2002) arXiv:hep-th/0108147.

[57] E. C. Vagenas, Phys. Lett. B 533, 302 (2002) arXiv:hep-th/0109108.

[58] E. C. Vagenas, Phys. Lett. B 559, 65 (2003) arXiv:hep-th/0209185].

[59] M. R. Setare and E. C. Vagenas, Phys. Lett. B 584, 127 (2004) arXiv:hep-th/0309092.

[60] M. R. Setare and E. C. Vagenas, Int. J. Mod. Phys. A 20, 7219 (2005) arXiv:hep-th/0405186.

[61] A. J. M. Medved and E. C. Vagenas, Mod. Phys. Lett. A 20, 1723 (2005) arXiv:gr-qc/0505015].

[62] R. Banerjee, B. R. Majhi and E. C. Vagenas, Phys. Lett. B 686, 279 (2010) arXiv:0907.4271 [hep-th]].

[63] R. Banerjee and S. K. Modak, JHEP 0911, 073 (2009) [arXiv:0908.2346 [hep-th]].

[64] D. Roy, Phys. Lett. B 681, 185 (2009) [arXiv:0908.3149 [hep-th]].

[65] L. C. Zhang, H. F. Li, Y. Q. Wu and R. Zhao, Int. J. Theor. Phys. 49, 1587 (2010).

[66] J. R. Ren, P. J. Mao, R. Li, T. Zhu and L. Y. Jia, Mod. Phys. Lett. A 25, 2167 (2010).

[67] B. R. Majhi, Phys. Lett. B 686, 49 (2010) [arXiv:0911.3239 [hep-th]]. 
[68] R. Banerjee, B. R. Majhi and E. C. Vagenas, Europhys. Lett. 92, 20001 (2010) arXiv:1005.1499 [hep-th]].

[69] R. Banerjee, C. Kiefer and B. R. Majhi, Phys. Rev. D 82, 044013 (2010) arXiv:1005.2264 $[\mathrm{gr}-\mathrm{qc}]]$.

[70] A. Yale and R. B. Mann, Phys. Lett. B673, 168-172 (2009). [arXiv:0808.2820 [gr-qc]].

[71] A. Yale, arXiv:1012.2114 [gr-qc].

[72] A. Yale, arXiv:1012.3165 [gr-qc].

[73] J. Y. Zhang and Z. Zhao, Mod. Phys. Lett. A 20, 1673 (2005).

[74] J. Y. Zhang and Z. Zhao, Phys. Lett. B 618, 14 (2005).

[75] Q. Q. Jiang, S. Q. Wu and X. Cai, Phys. Rev. D 73, 064003 (2006) [Erratum-ibid. D 73, 069902 (2006)] arXiv:hep-th/0512351.

[76] D. Chen and S. Yang, Mod. Phys. Lett. A 22, 2611 (2007).

[77] K. Umetsu, arXiv:1003.5534 [hep-th].

[78] K. Umetsu, Phys. Lett. B 692, 61 (2010) [arXiv:1007.1823 [hep-th]].

[79] S. Iso, H. Umetsu and F. Wilczek, Phys. Rev. Lett. 96, 151302 (2006) arXiv:hep-th/0602146.

[80] S. Iso, H. Umetsu and F. Wilczek, Phys. Rev. D 74, 044017 (2006) arXiv:hep-th/0606018.

[81] S. Iso, T. Morita and H. Umetsu, JHEP 0704, 068 (2007) arXiv:hep-th/0612286.

[82] K. Umetsu, Prog. Theor. Phys. 119, 849 (2008) arXiv:0804.0963 [hep-th]].

[83] K. Lin and S. Yang, Int. J. Theor. Phys. 49, 927 (2010) and references therein.

[84] P. Dobiasch and D. Maison, Gen. Rel. Grav. 14, 231 (1982).

[85] G. W. Gibbons and D. L. Wiltshire, Annals Phys. 167, 201 (1986) [Erratum-ibid. 176, 393 (1987)].

[86] H. Ishihara and K. Matsuno, Prog. Theor. Phys. 116, 417 (2006) arXiv:hep-th/0510094.

[87] R. G. Cai, L. M. Cao and N. Ohta, Phys. Lett. B 639, 354 (2006) arXiv:hep-th/0603197.

[88] Y. Kurita and H. Ishihara, Class. Quant. Grav. 24, 4525 (2007) [arXiv:0705.0307 [hep-th]].

[89] Y. Kurita and H. Ishihara, Class. Quant. Grav. 25, 085006 (2008) [arXiv:0801.2842 [hep-th]].

[90] H. Ishihara and J. Soda, Phys. Rev. D 76, 064022 (2007) arXiv:hep-th/0702180.

[91] S. Chen, B. Wang and R. K. Su, Phys. Rev. D 77, 024039 (2008) [arXiv:0710.3240 [hep-th]].

[92] S. W. Wei, R. Li, Y. X. Liu and J. R. Ren, Eur. Phys. J. C 65, 281 (2010) arXiv:0901.2614 [hep-th]].

[93] H. Ishihara, M. Kimura, R. A. Konoplya, K. Murata, J. Soda and A. Zhidenko, Phys. Rev. 
D 77, 084019 (2008) [arXiv:0802.0655 [hep-th]].

[94] X. He, B. Wang, S. Chen, R. G. Cai and C. Y. Lin, Phys. Lett. B 665, 392 (2008) arXiv:0802.2449 [hep-th]].

[95] X. He, B. Wang and S. Chen, Phys. Rev. D 79, 084005 (2009) arXiv:0811.2322 [gr-qc]].

[96] M. Kimura, K. Murata, H. Ishihara and J. Soda, Phys. Rev. D 77, 064015 (2008) arXiv:0712.4202 [hep-th]].

[97] R. Nishikawa and M. Kimura, Class. Quant. Grav. 27, 215020 (2010) arXiv:1005.1367] [hepth]].

[98] K. Matsuno and H. Ishihara, Phys. Rev. D 80, 104037 (2009) arXiv:0909.0134 [hep-th]].

[99] Y. Liu, S. Chen and J. Jing, Phys. Rev. D 81, 124017 (2010) [arXiv:1003.1429 [gr-qc]].

[100] F. R. Tangherlini, Nuovo Cim. 27, 636 (1963).

[101] R. Banerjee and B. R. Majhi, Phys. Lett. B 675, 243 (2009) arXiv:0903.0250 [hep-th]].

[102] K. Umetsu, Int. J. Mod. Phys. A 25, 4123 (2010) [arXiv:0907.1420 [hep-th]]. 